\title{
Morphological and Molecular Characterization of Complete Panicle Emergence Mutant Lines for Assessing Genetic Relatedness
}

\author{
B. Suneel ${ }^{1}$, G. S. Laha ${ }^{1}$, A. P. Padmakumari ${ }^{1}$, R. M. Sundaram ${ }^{1}$, \\ L. V. Subba Rao', P. Sudhakar ${ }^{2}$, A. Krishna Satya ${ }^{2}$ and M. S. Madhav ${ }^{\text {* }}$ \\ ${ }^{1}$ ICAR-Indian Institute of Rice Research, Hyderabad, India \\ ${ }^{2}$ Department of Biotechnology, Acharya Nagarjuna University, Guntur, India \\ *Corresponding author
}

\section{A B S T R A C T}

\begin{tabular}{|c|}
\hline Keywords \\
\hline $\begin{array}{l}\text { Samba Mahsuri, } \\
\text { Complete panicle } \\
\text { emergence, DUS, } \\
\text { morphological } \\
\text { traits, Mutants, } \\
\text { Correlation, } \\
\text { Variation, PPV \& } \\
\text { FRA Act }\end{array}$ \\
\hline Article Info \\
\hline $\begin{array}{l}\text { Accepted: } \\
\text { 04 August } 2020 \\
\text { Available Online: } \\
\text { 10 September } 2020\end{array}$ \\
\hline
\end{tabular}

\section{Keywords}

Samba Mahsuri, Complete panicle emergence, DUS,

Correlation, Variation, PPV \&

\section{Article Info}

04 August 2020

10 September 2020

\begin{abstract}
Morphological characterization of thirteen promising CPE (complete panicle emergence) mutants of Samba Mahsuri was done using thirty four agro-morphological traits by following DUS (Distinctiveness, Uniformity and Stability) guide lines. There was no variation observed in twenty two characters while in 12 characters there was variation among the individual mutants. Among all the traits, Culm attitude showed higher variation but there was no variation in the panicle emergence. Genetic relatedness among the mutants was assessed by the hyper polymorphic SSR markers. Correlation of phenotypic and genotypic data among mutants revealed that three mutants (CPE-1, CPE-9 \& CPE-10) showed high genetic similarity with wild type $(95 \%, 98.33 \% \& 96.66 \%)$ as well as negligible variation in tested morphological characters. Whereas two mutants (CPE-12 \& 13) showed more variation in morphological characters as well as less genetic similarity (86.66 \& $86.66 \%$ ) with wild type was observed. But all the genotypes used in this study exhibits complete panicle emergence, which is differ from the wild type parent. This detailed characterization of Samba Mahsuri CPE mutants is very important for rice breeding from the standpoint of selection and conservation of different mutants for further utilization in crop improvement programmes and also to seek protection under PPV\&FR Act, 2001 of India.
\end{abstract}

\section{Introduction}

Rice (Oryza sativa L.) is the world's most important staple food crop for more than 50\% of world's population and also a model plant that has attracted broad interests in basic and applied research (Sasaki et al., 2002, Mohapatra et al., 2004, Singh and Singh, 2008). More than $90 \%$ of rice consumption is in Asian countries and considered as 'Rice
Basket' of the world (Komala et al., 2017). To meet the need of ever growing population of the world, rice production need to increase. To overcome the yield ceiling issue, diverse genetic resources including near-isogenic lines, land races, wild spices and mutant populations are being used (Jian-Li Wu et al., 2005). Among these, mutant stocks also used to determine the function of genes and their biochemical and metabolic pathways (Jian-Li 
$\mathrm{Wu}$ et al., 2005). Many national and international research groups are actively working on the production of rice mutants (Hirochika et al., 2004; Leung and an, 2004). The most popular approach is induced mutagenesis and many important varieties of crops belongs to rice, cotton, rapeseed, sunflower, sesame, grapefruit, banana, ornamentals with desired characters were released through mutation breeding and they played a major role in global economic impact (Subodh K. Datta 2012). As per the FAO/IAEA Mutant Varieties Database, 3200 varieties have been released worldwide for cultivation in different countries through mutation breeding. Almost 700 mutant rice varieties were produced through mutation breeding, and maximum mutants were developed in cereals followed by barley, wheat, maize, durum wheat, oat, millet, sorghum and rye (Suprasanna et al., 2015). Ethyl methane sulphonate particularly generates point mutations and it is effective than physical mutagens. EMS proficiently enhances the chemical modifications of nucleotides within the rice genome and it mainly produces random point mutations and the mutants were $\mathrm{C} / \mathrm{G}$ to $\mathrm{T} / \mathrm{A}$ transitions type (Reddy 2000; Koornneeff et al., 1982; Rao 1977). EMS generates gene mutation in high frequency and chromosome aberration in low frequency (Van Harten1998; Lai et al., 2004). EMS induced mutagenesis was chosen for creation of large variants in the popular variety, Samba Mahsuri (Gopi et al., 2013). Characterization of mutant in terms of genetic as well as in morphological characters through distinctiveness, uniformity and stability (DUS) guidelines depicted in PPV \& FRA in 2001 is very much necessary for the protection of material as well as use in the breeding programmes. Hence, in the present study the mutants which showed the complete panicle emergence was selected for molecular and morphological characterization.

\section{Materials and Methods}

Thirteen complete panicle emergence Samba Mahsuri mutant lines of rice (Suneel et al., 2020) (Table 1) were grown in a RCBD (randomized complete block design) with two replications at IIRR farm, Rajendranagar, Hyderabad, situated at $17.53 \mathrm{~N}$ latitude and 78.27E longitude, $545 \mathrm{~m}$ altitude, with a mean temperature of $31.2^{\circ} \mathrm{C}$ and mean annual precipitation of $988.3 \mathrm{~mm}$. The material was grown with wild type (BPT-5204) in two replications during Kharif 2016. Thirteen CPE genotypes seed was sowed on nursery beds and 25-30 days old seedlings were transplanted into the field. The plant spacing was $15 \mathrm{~cm}$ to $20 \mathrm{~cm}$ with density of one hill. The recommended packages of practices were followed to raise the crop in the field and to avoid the interspecific competition in between the plants regular hand weeding was embarked to free the plant. Five plants were selected randomly to take the observations. Selected plants of each genotype were subjected to thirty four morphological traits. Among thirty four (18 essential and 16 additional) quantitative traits, were assessed visually as per the DUS test guidelines by UPOV PPV \& FR Act, 2001 for rice. The observations of selected genotype for different characteristics were recorded at different stages of growth.

\section{DNA Extraction}

Genomic DNA of CPE individuals and their respective wild type were isolated using modified cetyl trimethyl ammonium bromide (CTAB) method (Devi et al., 2020).

\section{SSR amplification}

SSR amplification the veriti PCR system (M/S Applied Bio Systems, USA) was used with following buffer composition. DNA template $(30 \mathrm{ng} / \mu \mathrm{L}), 2 \mu \mathrm{L}$ of $10 \mathrm{x}$ PCR buffer 
(kappa), $0.5 \mu \mathrm{L}$ of forward and reverse primers each $(5 \mathrm{pM} / \mu \mathrm{L}), 1 \mu \mathrm{L}$ of dNTP's ( $2.5 \mathrm{mM}$ conc) and $0.1 \mu \mathrm{L}$ of taq-polymerase (kappa,5U) and the final reaction volume was made up to $20 \mu \mathrm{L}$ with nuclease free $\mathrm{H}_{2} 0$.

\section{SSR allele scoring and data analysis}

Genomic similarity of thirteen CPE promising mutants was carried out with wild type using randomly selected 60 SSR markers spread over 12 chromosomes. The presence or absence of SSR fragment in each mutant genotype was recorded. The SSR amplicons appearing without ambiguity were scored as 1 (present) and 0 (absent) for each primer. The amplified PCR product size was calculated on the basis of its mobility relative to molecular mass of marker (100 bp DNA ladder).

\section{Results and Discussion}

A total of $13 \mathrm{M}_{8}$ mutant lines (Table 1) along with wild type (BPT-5204) were taken for characterization using 34 DUS characters (Table 2). The Samba Mahsuri each CPE mutant line showed distinctiveness in few morphological traits. Earlier researchers reported the distinctiveness in the morphological traits of rice landraces (Rao et al., (2013); Tirkey et al., 2013); Mondal et al., (2014); Manjunatha et al., 2016); Kalyan et al., (2017); Komala et al., (2017) and Umarani et al., (2017). Quantitative characters of thirteen CPE mutants are presented in Table 4. Among 13 mutants, 7 mutants (CPE-1, 2, 3, 8, 9, 10 and 11) showed dark like wild type, 5 mutants (CPE- 4,5,7,12 $\&$ 13) showed medium and 1 mutant (CPE-6) showed light green colour of DUS trait leaf intensity of green colour. Pubescence of blade surface was weak in 2 mutants (CPE-5 \& 8), medium in 6 mutants (CPE-1, 2, 3, 4, 9 \& 10) which was like wild type, and strong in 5 mutants (CPE-6, 7, 11, 12 \& 13). A total of seven mutant genotypes were distinct from the wild type in the pubescence of blade surface. Culm: attitude was erect in 1 mutant (CPE-6), semi erect in 7 mutants (CPE-1, 2, $3,4,9,10 \& 11)$ which was like wild type, open in 2 mutants (CPE-12 \&13) and spreading in 3 mutants (CPE-5, $7 \& 8$ ). While coming to the flag leaf attitude of blade (early observation) among CPE mutants, erect type of flag leaf attitude (early) was observed in CPE-1,2,3,7,9,10 \& 11 like wild type, and semi erect type was observed in CPE-4, 5, 6, $8,12 \& 13$ which is distinct from the wild type. Density of pubescence of lemma showed weak in 1 mutant, 7 mutants showed medium like wild type and 5 mutants with strong pubescence. Stem thickness was medium $(<0.40-0.55 \mathrm{~cm})$ in 7 mutants (CPE$1,2,3,4,8,9, \& 10)$ which was like wild type, thin $(<0.40 \mathrm{~cm})$ in 3 mutants (CPE-5, 6 $\& 7)$, and thick stem $(>0.55 \mathrm{~cm})$ was observed in 3 mutants (CPE-11, $12 \&$ 13). While coming to the Flag Leaf attitude (late observation) CPE-1, 3, 5, 6, 7, 9, 10, 11, 12 \& 13 were showed semi erect type like wild type and CPE-2, 4 \& 8 exhibits horizontal type, which is distinct from wild type. With respect to panicle characters 1 mutant (CPE-2) were of semi straight, 7 mutants (CPE-1, 3, 6, 7, 9, $10 \& 11)$ exhibits deflexed type like wild type, and 5 mutants (CPE-4, 5, 8, 12 \& 13) exhibits drooping type. Lemma \& Palea: colour was straw in 8 mutants (CPE-1, 2, 3, 5, 6, 7, $9 \& 10$ ) and 5 mutants (CPE-4, 8, 11, 12 \&13) showed brown furrows on straw, which is distinct from wild type (Fig. 1).

Panicle: Secondary branching was strong in 10 mutants (CPE-1, 2, 3, 4, 5, 6, 7, 8, 9 \& 10) which was like wild type and clustered in 3 mutants (CPE-11, $12 \& 13$ ). While coming to the panicle: attitude of branches 4 mutants (CPE-1, 9, 10 \& 11) were showed erect to semi erect type like wild type, 5 mutants (CPE-3, 4, 7, 12 \& 13) were showed semi erect type and 4 mutants (CPE-2, 5, 6 \& 8) were showed semi erect to spreading type 
which is differ from the wild type. The character Leaf: Senescence was early in 2 mutants (CPE-5 \& 6), medium in 6 mutants (CPE-1, 3, 4, 7, 9 \&10) and late in 5 mutants (CPE-2, 9, 11, 12 \& 13).

There was no variation were observed with respect to coleoptile: colour was colourless in all mutants. Basal leaf: sheath colour was green in all mutants. Leaf: anthocyanin colouration and leaf sheath anthocyanin colouration was absent in all mutants. All CPE mutants have leaf auricles and the anthocyanin colouration of auricles was colourless or absent in all mutants. Leaf: Collar was present in thirteen CPE mutants and anthocyanin colouration of Collar was absent in all. Leaf: Ligule was present in all and shape of the ligule was split in all mutants with white in colour. It is important to note that no mutant line exhibited male sterility. Lemma: Anthocyanin colouration of keel and anthocyanin colouration of area below apex was absent in all lines. Spikelet: Colour of Stigma was white in colour in all mutant genotypes. Stem: Intensity of anthocyanin colouration of nodes was not observed. Stem: anthocyanin colouration of internodes was absent in all CPE individuals. Spikelet colour of tip of lemma was yellowish in all mutants. Panicle: Awns was absent in thirteen mutants and panicle: secondary branching was present in all. Sterile: Lemma colour was straw in all mutant genotypes.

\section{Molecular characterization}

Genetic diversity assessment is not only important for crop improvement but also for efficient management and protection of germplasm resources (Yogendra Singh, 2011; Singh and Singh, 2008) and molecular approaches are widely used by researchers time to time (Singh et al., 2013; Koutu et al., 2017; Koutu et al., 2019). Genetic relatedness among the mutants was assessed by the hyper polymorphic SSR markers. Correlation of phenotypic and genotypic data among mutants revealed that three mutants (CPE-1, CPE-9 \& CPE-10) showed high genetic similarity with wild type $(95 \%, 98.33 \%$ \& 96.66; Table 3) as well as negligible variation in tested morphological characters. Whereas two mutants (CPE-12 \& 13) showed more variation in morphological characters as well as less genetic similarity (86.66 \& $86.66 \%)$ with wild type was observed.

Table.1 List of Samba Mahsuri CPE mutants used in this present study

\begin{tabular}{|c|c|}
\hline S. No & Mutant \\
\hline $\mathbf{1}$ & CPE-1 \\
\hline $\mathbf{2}$ & CPE-2 \\
\hline $\mathbf{3}$ & CPE-3 \\
\hline $\mathbf{4}$ & CPE-4 \\
\hline $\mathbf{5}$ & CPE-5 \\
\hline $\mathbf{6}$ & CPE-6 \\
\hline $\mathbf{7}$ & CPE-7 \\
\hline $\mathbf{8}$ & CPE-8 \\
\hline $\mathbf{9}$ & CPE-9 \\
\hline $\mathbf{1 0}$ & CPE-10 \\
\hline $\mathbf{1 1}$ & CPE-11 \\
\hline $\mathbf{1 2}$ & CPE-12 \\
\hline $\mathbf{1 3}$ & CPE-13 \\
\hline
\end{tabular}


Table.2 Details of 34 DUS characters considered in the present study

\begin{tabular}{|c|c|c|c|c|}
\hline S. No & Characteristics & States & Note & Stage of observation \\
\hline 1 & Coleoptile: Colour & $\begin{array}{l}\text { Colourless } \\
\text { Green } \\
\text { Purple }\end{array}$ & $\begin{array}{l}1 \\
2 \\
3\end{array}$ & 2-3days after sowing \\
\hline$* 2$ & Basal leaf: Sheath colour & $\begin{array}{l}\text { Green } \\
\text { Light purple } \\
\text { Purple lines } \\
\text { Uniform purple }\end{array}$ & $\begin{array}{l}1 \\
2 \\
3 \\
4\end{array}$ & Booting \\
\hline 3 & Leaf: Intensity of green colour & $\begin{array}{l}\text { Light } \\
\text { Medium } \\
\text { Dark }\end{array}$ & $\begin{array}{l}3 \\
5 \\
7\end{array}$ & Booting \\
\hline S. No & Characteristics & States & Note & Stage of observation \\
\hline 4 & $\begin{array}{l}\text { Leaf: Anthocyanin } \\
\text { colouration }\end{array}$ & $\begin{array}{l}\text { Absent } \\
\text { Present }\end{array}$ & $\begin{array}{l}1 \\
9\end{array}$ & Booting \\
\hline 5 & $\begin{array}{l}\text { Leaf sheath: Anthocyanin } \\
\text { colouration }\end{array}$ & $\begin{array}{l}\text { Absent } \\
\text { Present }\end{array}$ & $\begin{array}{l}1 \\
9\end{array}$ & Booting \\
\hline$* 6$ & $\begin{array}{l}\text { Leaf: Pubescence of blade } \\
\text { surface }\end{array}$ & $\begin{array}{l}\text { Absent } \\
\text { Weak } \\
\text { Medium } \\
\text { Strong } \\
\text { Very strong }\end{array}$ & $\begin{array}{l}1 \\
3 \\
5 \\
7 \\
9\end{array}$ & Booting \\
\hline$* 7$ & Leaf: Auricles & $\begin{array}{l}\text { Absent } \\
\text { Present }\end{array}$ & $\begin{array}{l}1 \\
9\end{array}$ & Booting \\
\hline$* 8$ & $\begin{array}{l}\text { Leaf: Anthocyanin } \\
\text { colouration of auricles }\end{array}$ & $\begin{array}{l}\text { Colourless } \\
\text { Light purple } \\
\text { Purple }\end{array}$ & $\begin{array}{l}1 \\
2 \\
3\end{array}$ & Booting \\
\hline 9 & Leaf: Collar & $\begin{array}{l}\text { Absent } \\
\text { Present }\end{array}$ & $\begin{array}{l}1 \\
9\end{array}$ & Booting \\
\hline 10 & $\begin{array}{l}\text { Leaf: Anthocyanin } \\
\text { colouration of Collar }\end{array}$ & $\begin{array}{l}\text { Absent } \\
\text { Present }\end{array}$ & $\begin{array}{l}1 \\
9\end{array}$ & Booting \\
\hline 11 & Leaf: Ligule & $\begin{array}{l}\text { Absent } \\
\text { Present }\end{array}$ & $\begin{array}{l}1 \\
9\end{array}$ & Booting \\
\hline$* 12$ & Leaf: Shape of ligule & $\begin{array}{l}\text { Truncate } \\
\text { Acute } \\
\text { Split }\end{array}$ & $\begin{array}{l}1 \\
2 \\
3\end{array}$ & Booting \\
\hline 13 & Leaf: Colour of ligule & $\begin{array}{l}\text { White } \\
\text { Light purple } \\
\text { Purple }\end{array}$ & $\begin{array}{l}1 \\
2 \\
3\end{array}$ & Booting \\
\hline 14 & Culm: Attitude & $\begin{array}{l}\text { Erect } \\
\text { Semi-erect } \\
\text { Open } \\
\text { Spreading }\end{array}$ & $\begin{array}{l}1 \\
3 \\
5 \\
7\end{array}$ & Booting \\
\hline$* 15$ & $\begin{array}{l}\text { Flag leaf: Attitude of blade } \\
\text { (early observation) }\end{array}$ & $\begin{array}{l}\text { Erect } \\
\text { Semi-erect } \\
\text { Horizontal } \\
\text { Drooping }\end{array}$ & $\begin{array}{l}1 \\
3 \\
5 \\
7\end{array}$ & Beginning of anthesis \\
\hline$* 16$ & $\begin{array}{l}\text { Spikelet: Density of } \\
\text { pubescence of lemma }\end{array}$ & $\begin{array}{l}\text { Absent } \\
\text { Weak } \\
\text { Medium } \\
\text { Strong } \\
\text { Very strong }\end{array}$ & $\begin{array}{l}1 \\
3 \\
5 \\
7 \\
9\end{array}$ & Beginning of anthesis \\
\hline
\end{tabular}




\begin{tabular}{|c|c|c|c|c|}
\hline S. No & Characteristics & States & Note & Stage of observation \\
\hline 17 & Male sterility & $\begin{array}{l}\text { Absent } \\
\text { Present }\end{array}$ & $\begin{array}{l}1 \\
9\end{array}$ & Anthesis half way \\
\hline 18 & $\begin{array}{l}\text { Lemma: Anthocyanin } \\
\text { colouration of keel }\end{array}$ & $\begin{array}{l}\text { Absent or very } \\
\text { weak } \\
\text { Weak } \\
\text { Medium } \\
\text { Strong } \\
\text { Very strong }\end{array}$ & $\begin{array}{l}1 \\
3 \\
5 \\
7 \\
9\end{array}$ & Anthesis half way \\
\hline$* 19$ & $\begin{array}{l}\text { Lemma: Anthocyanin } \\
\text { colouration of area below } \\
\text { apex }\end{array}$ & $\begin{array}{l}\text { Absent } \\
\text { Weak } \\
\text { Medium } \\
\text { Strong } \\
\text { Very strong }\end{array}$ & $\begin{array}{l}1 \\
3 \\
5 \\
7 \\
9\end{array}$ & Anthesis half way \\
\hline 20 & $\begin{array}{l}\text { Lemma: Anthocyanin } \\
\text { colouration of apex }\end{array}$ & $\begin{array}{l}\text { Absent } \\
\text { Weak } \\
\text { Medium } \\
\text { Strong } \\
\text { Very strong }\end{array}$ & $\begin{array}{l}1 \\
3 \\
5 \\
7 \\
9\end{array}$ & Anthesis half way \\
\hline$* 21$ & Spikelet: Colour of stigma & $\begin{array}{l}\text { White } \\
\text { Light green } \\
\text { Yellow } \\
\text { Light purple } \\
\text { Purple }\end{array}$ & $\begin{array}{l}1 \\
2 \\
3 \\
4 \\
5\end{array}$ & Anthesis half way \\
\hline 22 & Stem: Thickness & $\begin{array}{l}\text { Thin }(<0.40 \mathrm{~cm}) \\
\text { Medium }(0.40- \\
0.55) \\
\text { Thick }(>0.55 \mathrm{~cm})\end{array}$ & $\begin{array}{l}3 \\
5 \\
7\end{array}$ & Milky stage of plant \\
\hline$* 23$ & $\begin{array}{l}\text { Stem: Anthocyanin } \\
\text { colouration of nodes }\end{array}$ & $\begin{array}{l}\text { Absent } \\
\text { Present }\end{array}$ & $\begin{array}{l}1 \\
9\end{array}$ & Milky stage of plant \\
\hline 24 & $\begin{array}{l}\text { Stem: Anthocyanin } \\
\text { colouration of internodes }\end{array}$ & $\begin{array}{l}\text { Absent } \\
\text { Present }\end{array}$ & $\begin{array}{l}1 \\
9\end{array}$ & Milky stage of plant \\
\hline$* 25$ & $\begin{array}{l}\text { Flag leaf: Attitude of blade } \\
\text { (late observation) }\end{array}$ & $\begin{array}{l}\text { Erect } \\
\text { Semi-erect } \\
\text { Horizontal } \\
\text { Deflexed }\end{array}$ & $\begin{array}{l}1 \\
3 \\
5 \\
7\end{array}$ & Maturity stage \\
\hline$* 26$ & $\begin{array}{l}\text { Panicle: Curvature of main } \\
\text { axis }\end{array}$ & $\begin{array}{l}\text { Straight } \\
\text { Semi straight } \\
\text { Deflexed } \\
\text { Drooping }\end{array}$ & $\begin{array}{l}1 \\
3 \\
5 \\
7\end{array}$ & Maturity stage \\
\hline$* 27$ & $\begin{array}{l}\text { Spikelet: Colour of tip of } \\
\text { lemma }\end{array}$ & $\begin{array}{l}\text { White } \\
\text { Yellowish } \\
\text { Brown } \\
\text { Red } \\
\text { Purple } \\
\text { Black }\end{array}$ & $\begin{array}{l}1 \\
2 \\
3 \\
4 \\
5 \\
6\end{array}$ & Maturity stage \\
\hline 28 & Lemma and Palea: Colour & $\begin{array}{l}\text { Straw } \\
\text { Gold and Gold } \\
\text { furrows on straw } \\
\text { back ground } \\
\text { Brown spots on } \\
\text { straw } \\
\text { Brown furrows } \\
\text { on straw } \\
\text { Brown(tawny) }\end{array}$ & $\begin{array}{l}1 \\
2 \\
3 \\
4 \\
\\
5 \\
6\end{array}$ & Maturity stage \\
\hline
\end{tabular}




\begin{tabular}{|c|c|c|c|c|}
\hline & & $\begin{array}{l}\text { Reddish to light } \\
\text { purple } \\
\text { Furrows on straw } \\
\text { Purple } \\
\text { Black }\end{array}$ & $\begin{array}{l}7 \\
8 \\
9\end{array}$ & \\
\hline$* 29$ & Panicle: Awns & $\begin{array}{l}\text { Absent } \\
\text { Present }\end{array}$ & $\begin{array}{l}1 \\
9\end{array}$ & Maturity stage \\
\hline 30 & $\begin{array}{l}\text { Panicle: Presence of } \\
\text { secondary branching }\end{array}$ & $\begin{array}{l}\text { Absent } \\
\text { Present }\end{array}$ & $\begin{array}{l}1 \\
9\end{array}$ & Maturity stage \\
\hline 31 & Panicle: Secondary branching & $\begin{array}{l}\text { Weak } \\
\text { Strong } \\
\text { Clustered }\end{array}$ & $\begin{array}{l}1 \\
2 \\
3\end{array}$ & Maturity stage \\
\hline 32 & Panicle: Attitude of branches & $\begin{array}{l}\text { Erect } \\
\text { Erect to semi } \\
\text { erect } \\
\text { Semi erect } \\
\text { Semi erect to } \\
\text { spreading } \\
\text { Spreading }\end{array}$ & $\begin{array}{l}1 \\
3 \\
\\
5 \\
\\
7 \\
9\end{array}$ & Maturity stage \\
\hline 33 & Leaf: Senescence & $\begin{array}{l}\text { Early } \\
\text { Medium } \\
\text { Late }\end{array}$ & $\begin{array}{l}3 \\
5 \\
7\end{array}$ & Maturity stage \\
\hline$* 34$ & Sterile lemma: Colour & $\begin{array}{l}\text { Straw } \\
\text { Gold } \\
\text { Red } \\
\text { Purple }\end{array}$ & $\begin{array}{l}1 \\
2 \\
3 \\
4\end{array}$ & Maturity stage \\
\hline
\end{tabular}

Table.3 Assessment of similarity of CPE mutants with wild type

\begin{tabular}{|c|c|c|c|c|}
\hline S.no & Genotype & $\begin{array}{c}\text { No. of markers } \\
\text { tested }\end{array}$ & $\begin{array}{c}\text { No. of markers } \\
\text { monomorphic }\end{array}$ & $\begin{array}{c}\text { \% similarity with } \\
\text { wild type }\end{array}$ \\
\hline $\mathbf{1}$ & CPE-1 & 60 & 57 & 95.00 \\
\hline $\mathbf{2}$ & CPE-2 & 60 & 54 & 90.00 \\
\hline $\mathbf{3}$ & CPE-3 & 60 & 55 & 91.66 \\
\hline $\mathbf{4}$ & CPE-4 & 60 & 54 & 90.00 \\
\hline $\mathbf{5}$ & CPE-5 & 60 & 55 & 91.66 \\
\hline $\mathbf{6}$ & CPE-6 & 60 & 54 & 90.00 \\
\hline $\mathbf{7}$ & CPE-7 & 60 & 56 & 93.33 \\
\hline $\mathbf{8}$ & CPE-8 & 60 & 53 & 88.33 \\
\hline $\mathbf{9}$ & CPE-9 & 60 & 59 & 98.33 \\
\hline $\mathbf{1 0}$ & CPE-10 & 60 & 58 & 96.66 \\
\hline $\mathbf{1 1}$ & CPE-11 & 60 & 55 & 91.66 \\
\hline $\mathbf{1 2}$ & CPE-12 & 60 & 52 & 86.66 \\
\hline $\mathbf{1 3}$ & CPE-13 & 60 & 52 & 86.66 \\
\hline
\end{tabular}


Table.4 Classification of CPE mutants based on DUS characters

\begin{tabular}{|c|c|c|c|c|c|c|c|c|c|c|c|c|c|c|c|}
\hline S.no & Characteristics & $\begin{array}{c}\text { CPE- } \\
1\end{array}$ & $\begin{array}{c}\text { CPE- } \\
2\end{array}$ & $\begin{array}{c}\text { CPE- } \\
3\end{array}$ & $\begin{array}{c}\text { CPE- } \\
4\end{array}$ & $\begin{array}{l}\text { CPE- } \\
5\end{array}$ & $\begin{array}{c}\text { CPE- } \\
6\end{array}$ & $\begin{array}{c}\text { CPE- } \\
7\end{array}$ & $\begin{array}{c}\text { CPE- } \\
8\end{array}$ & $\begin{array}{c}\text { CPE- } \\
9\end{array}$ & $\begin{array}{c}\text { CPE- } \\
10\end{array}$ & CPE11 & $\begin{array}{l}\text { CPE- } \\
12\end{array}$ & $\begin{array}{c}\text { CPE- } \\
13\end{array}$ & $\begin{array}{l}\text { BPT- } \\
5204\end{array}$ \\
\hline 1 & Coleoptile: Colour & 1 & 1 & 1 & 1 & 1 & 1 & 1 & 1 & 1 & 1 & 1 & 1 & 1 & $\mathbf{1}$ \\
\hline$* 2$ & Basal leaf: Sheath colour & 1 & 1 & 1 & 1 & 1 & 1 & 1 & 1 & 1 & 1 & 1 & 1 & 1 & 1 \\
\hline 3 & $\begin{array}{l}\text { Leaf: Intensity of green } \\
\text { colour }\end{array}$ & 7 & 7 & 7 & 5 & 5 & 3 & 5 & 7 & 7 & 7 & 7 & 5 & 5 & 7 \\
\hline 4 & $\begin{array}{l}\text { Leaf: Anthocyanin } \\
\text { colouration }\end{array}$ & 1 & 1 & 1 & 1 & 1 & 1 & 1 & 1 & 1 & 1 & 1 & 1 & 1 & 1 \\
\hline 5 & $\begin{array}{l}\text { Leaf sheath: Anthocyanin } \\
\text { colouration }\end{array}$ & 1 & 1 & 1 & 1 & 1 & 1 & 1 & 1 & 1 & 1 & 1 & 1 & 1 & 1 \\
\hline$* 6$ & $\begin{array}{l}\text { Leaf: Pubescence of blade } \\
\text { surface }\end{array}$ & 5 & 5 & 5 & 5 & 3 & 7 & 7 & 3 & 5 & 5 & 7 & 7 & 7 & 5 \\
\hline$* 7$ & Leaf: Auricles & 9 & 9 & 9 & 9 & 9 & 9 & 9 & 9 & 9 & 9 & 9 & 9 & 9 & 9 \\
\hline$* 8$ & $\begin{array}{l}\text { Leaf: Anthocyanin } \\
\text { colouration of auricles }\end{array}$ & 1 & 1 & 1 & 1 & 1 & 1 & 1 & 1 & 1 & 1 & 1 & 1 & 1 & $\mathbf{1}$ \\
\hline 9 & Leaf: Collar & 9 & 9 & 9 & 9 & 9 & 9 & 9 & 9 & 9 & 9 & 9 & 9 & 9 & 9 \\
\hline 10 & $\begin{array}{l}\text { Leaf: Anthocyanin } \\
\text { colouration of auricles }\end{array}$ & 1 & 1 & 1 & 1 & 1 & 1 & 1 & 1 & 1 & 1 & 1 & 1 & 1 & 1 \\
\hline 11 & Leaf: Ligule & 9 & 9 & 9 & 9 & 9 & 9 & 9 & 9 & 9 & 9 & 9 & 9 & 9 & 9 \\
\hline$* 12$ & Leaf: Shape of ligule & 3 & 3 & 3 & 3 & 3 & 3 & 3 & 3 & 3 & 3 & 3 & 3 & 3 & 3 \\
\hline$* 13$ & Leaf: Colour of ligule & 1 & 1 & 1 & 1 & 1 & 1 & 1 & 1 & 1 & 1 & 1 & 1 & 1 & 1 \\
\hline 14 & Culm: Attitude & 3 & 3 & 3 & 3 & 7 & 1 & 7 & 7 & 3 & 3 & 3 & 5 & 5 & 3 \\
\hline$* 15$ & $\begin{array}{l}\text { Flag leaf: Attitude of blade } \\
\text { (early observation) }\end{array}$ & 1 & 1 & 1 & 3 & 3 & 3 & 1 & 3 & 1 & 1 & 1 & 3 & 3 & 1 \\
\hline$* 16$ & $\begin{array}{l}\text { Spikelet: Density of } \\
\text { pubescence of lemma }\end{array}$ & 5 & 5 & 5 & 5 & 7 & 7 & 7 & 3 & 5 & 5 & 5 & 7 & 7 & 5 \\
\hline 17 & Male sterility & 1 & 1 & 1 & 1 & 1 & 1 & 1 & 1 & 1 & 1 & 1 & 1 & 1 & 1 \\
\hline 18 & Lemma: Anthocyanin & 1 & 1 & 1 & 1 & 1 & 1 & 1 & 1 & 1 & 1 & 1 & 1 & 1 & \\
\hline
\end{tabular}




\begin{tabular}{|c|c|c|c|c|c|c|c|c|c|c|c|c|c|c|c|}
\hline & colouration of keel & & & & & & & & & & & & & & 1 \\
\hline$* 19$ & $\begin{array}{l}\text { Lemma: Anthocyanin } \\
\text { colouration of area below } \\
\text { apex }\end{array}$ & 1 & 1 & 1 & 1 & 1 & 1 & 1 & 1 & 1 & 1 & 1 & 1 & 1 & 1 \\
\hline$* 20$ & $\begin{array}{l}\text { Lemma: Anthocyanin } \\
\text { colouration of apex }\end{array}$ & 1 & 1 & 1 & 1 & 1 & 1 & 1 & 1 & 1 & 1 & 1 & 1 & 1 & 1 \\
\hline$* 21$ & Spikelet: Colour of stigma & 1 & 1 & 1 & 1 & 1 & 1 & 1 & 1 & 1 & 1 & 1 & 1 & 1 & 1 \\
\hline 22 & Stem: Thickness & 5 & 5 & 5 & 5 & 3 & 3 & 3 & 5 & 5 & 5 & 7 & 7 & 7 & 5 \\
\hline$* 23$ & $\begin{array}{l}\text { Stem: Anthocyanin } \\
\text { colouration of nodes }\end{array}$ & 1 & 1 & 1 & 1 & 1 & 1 & 1 & 1 & 1 & 1 & 1 & 1 & 1 & 1 \\
\hline 24 & $\begin{array}{l}\text { Stem: Anthocyanin } \\
\text { colouration of internodes }\end{array}$ & 1 & 1 & 1 & 1 & 1 & 1 & 1 & 1 & 1 & 1 & 1 & 1 & 1 & 1 \\
\hline$* 25$ & $\begin{array}{l}\text { Flag leaf: Attitude of blade } \\
\text { (late observation) }\end{array}$ & 3 & 5 & 3 & 5 & 3 & 3 & 3 & 5 & 3 & 3 & 3 & 3 & 3 & 3 \\
\hline$* 26$ & $\begin{array}{l}\text { Panicle: Curvature of main } \\
\text { axis }\end{array}$ & 5 & 3 & 5 & 7 & 7 & 5 & 5 & 7 & 5 & 5 & 5 & 7 & 7 & 5 \\
\hline$* 27$ & $\begin{array}{l}\text { Spikelet: Colour of tip of } \\
\text { lemma }\end{array}$ & 2 & 2 & 2 & 2 & 2 & 2 & 2 & 2 & 2 & 2 & 2 & 2 & 2 & 2 \\
\hline 28 & Lemma and Palea: Colour & 1 & 1 & 1 & 4 & 1 & 1 & 1 & 4 & 1 & $\mathbf{1}$ & 4 & 4 & 4 & 1 \\
\hline$* 29$ & Panicle: Awns & 1 & 1 & 1 & 1 & 1 & 1 & 1 & 1 & 1 & 1 & 1 & 1 & 1 & 1 \\
\hline 30 & $\begin{array}{l}\text { Panicle: Presence of } \\
\text { secondary branching }\end{array}$ & 9 & 9 & 9 & 9 & 9 & 9 & 9 & 9 & 9 & 9 & 9 & 9 & 9 & 9 \\
\hline 31 & $\begin{array}{l}\text { Panicle: Secondary } \\
\text { branching }\end{array}$ & 2 & 2 & 2 & 2 & 2 & 2 & 2 & 2 & 2 & 2 & 3 & 3 & 3 & 2 \\
\hline$* 32$ & $\begin{array}{l}\text { Panicle: Attitude of } \\
\text { branches }\end{array}$ & 3 & 7 & 5 & 5 & 7 & 7 & 5 & 7 & 3 & 3 & 3 & 5 & 5 & 3 \\
\hline 33 & Leaf: Senescence & 5 & 7 & 5 & 5 & 3 & 3 & 5 & 7 & 5 & 5 & 7 & 7 & 7 & 5 \\
\hline$* 34$ & Sterile lemma: Colour & 1 & 1 & 1 & 1 & 1 & 1 & 1 & 1 & 1 & 1 & 1 & 1 & 1 & 1 \\
\hline
\end{tabular}

Bold denotes the variation in the trait

Note: (*) Essential characters 
Fig.1 variation in the DUS characters among CPE mutants: (a) Leaf: Auricles, (b) Leaf: Collar, (c) Leaf: Shape of Ligule, (d) Spikelet: colour of Stigma, (e) Stem: Thickness, (f) Stem: Nodes \& internodes, (g) Flag leaf: Attitude of blade (late observation), (h) Panicle: Curvature of main axis, (i)Lemma \& Palea: colour, (j) Spikelet: Colour of tip of lemma, (k) Panicle: Secondary branching

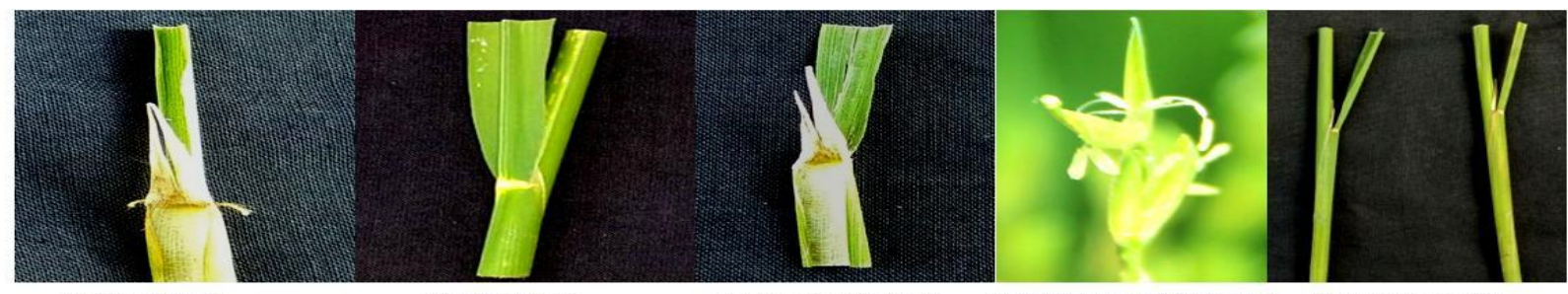

(a) Leaf: Auricle

(b) Leaf: Collar

(c) Leaf: Shape of ligule

(d) Spikelet: colour of Stigma

(e) Stem: Thickness
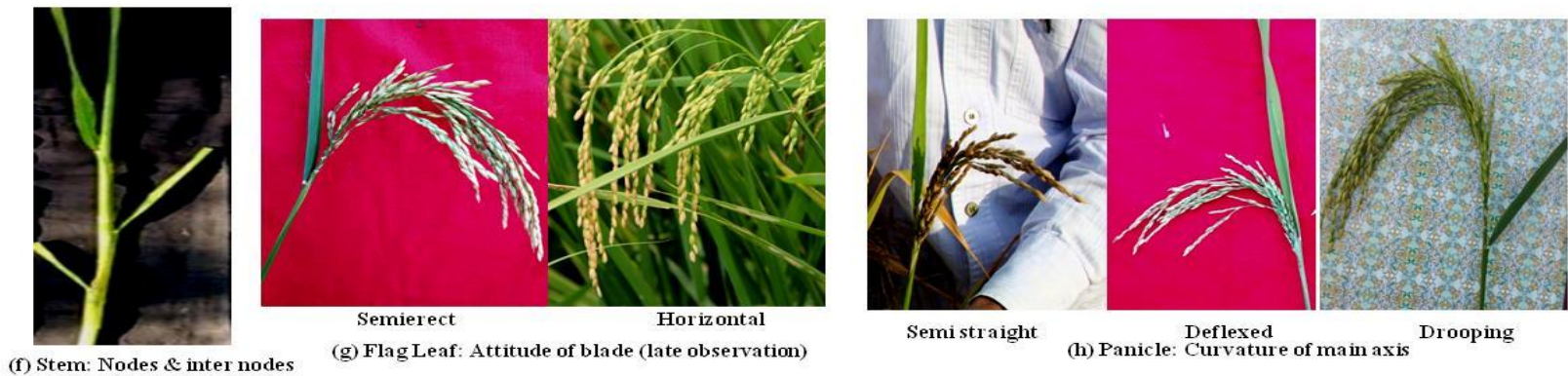

(h) Panicle: Curvature of main axis

Drooping

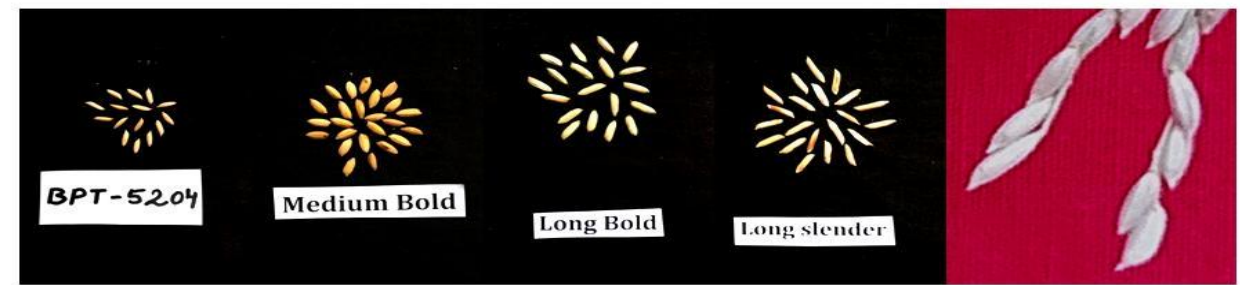

(i) Lemma \& palea colour in wild type \& mutants with different grain types

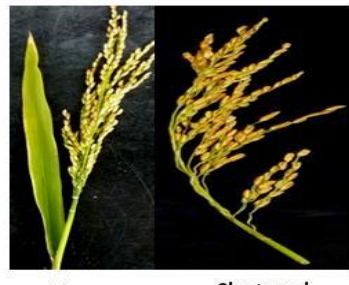

Strong Clustered
(k) Panicle: Secondarybranching
Among all mutant genotypes CPE-3 showed variation in only one character i.e. attitude of branches, which is differ from wild type. Among 34 quantitative characters Culm: attitude was showed higher variation. Among 13 CPE mutants CPE-2, CPE-4 \& CPE-6 showed $90 \%$ similarity with wild type, followed by CPE-3, CPE-5 \& CPE-11 (91.66\%), CPE-7 (93.33\%), and CPE-8 $(88.33 \%)$ respectively. But all the genotypes used in this study exhibits complete panicle emergence, which is differ from the wild type parent.

In conclusion, the present investigation, an effort was made to assess the $13 \mathrm{CPE}$ mutants of rice for genetic and morphological relatedness with the wild type. Uniformly spread 60 SSR markers were employed to assess their genetic diversity. CPE-1, 9 \& 10 showed high similarity with the wild type. The Samba Mahsuri CPE promising mutants identified in this study will be useful for plant breeders and researchers for getting productive and quality results in the plant breeding programs.

\section{Acknowledgements}

Authors are thankful to Council of Scientific and Industrial Research, India for financial support and ICAR-Indian Institute of Rice Research for providing facilities. 


\section{Competing interests}

Authors have declared that no competing interests exist.

\section{References}

GM Rao. Efficiency and effectiveness of gamma rays and EMS in rice. Cytologia. 1977; 42(3-4):443-450.

Gopi, J. Karteek, Md. Ershad, M.G. Gayathri, M. Milton, B.Suneel, A.P Padmakumari, G.S. Laha, M.S. Madhav, R.M. Sundaram, L.V. Subbarao, MR Vishnupriya, B C Viraktamat, and R V Sonti. 2013. Development of a mutagenized population and screening for resistance characteristics in the genetic background of the elite rice cultivar, Samba Mahsuri.

Hirochika, H., Guiderdoni, E., An, G., Hsing, Y., Eun, M.Y., Han, C.D., Upadhyaya, N., Ramachandran, S., Zhang, Q., Pereira, A., Sundaresan, V. and Leung, H. 2004. Rice mutant resources for gene discovery. Plant Mol. Biol. 54: 325-334.

Jian-Li Wu Chanjian Wu, Cailin Lei, Marietta Baraoidan, Alicia Bordeos, Ma. Reina Suzette Madamba, Marilou RamosPamplona, Ramil Mauleon, Arlett Portugal, Victor Jun Ulat, Richard Bruskiewich, Guoliang Wang, Jan Leach, Gurdev Khush and Hei Leung, Chemical- and irradiation-induced mutants of indica rice IR64 for forward and reverse genetics Plant Molecular Biology (2005) 59: 85-97.

Kalyan, B., Krishna, K. V. R. and Rao, S. L. V. (2017) DUS characterization for germplasm of rice. Int $\mathrm{J}$ Curr Microbiol App Sci., 6: 3480-3487.

Kamile Ulukapi and Ayse Gul Nasircilar Developments of Gamma Ray Application on Mutation Breeding
Studies in Recent Years, International Conference on Advances in Agricultural, Biological \& Environmental Sciences (AABES2015) July 22-23, 2015 London(UK).

Komala, N. T, Gurumurthy, R and Surendra, $\mathrm{P}$ Morphological Characterization of Advanced Lines of Rice (Oryza sativa L.) Derived from Swarna $x$ Ranbir Basmati at Seedling Stage.

Koornneeff M, Dellaert LWM, Van der Veen JH. EMS-and relation-induced mutation frequencies at individual loci in Arabidopsis thaliana (L.) Heynh. Mutation Research/Fundamental and Molecular Mechanisms of Mutagenesis. 1982; 93(1): 109-123.

Koutu G.K., A. Srivastava, M.S.Bhale, Yogendra Singh S.K. Singh and D. K. Mishra (2017). DNA Fingerprinting and Genetic Divergence analysis of Rice Cultivars using SSR markers. Indian Research Journal of Genetics and Bio Technology. 09 (03): 361368

Koutu G.K., A. Srivastava, Yogendra Singh and S. Tiwari (2019). Molecular Characterization and Genetic Diversity Assessment of Soybean Varieties using SSR Markers. International Journal of Current Microbiology and Applied Sciences. 08(04):173-182.

Leung, H. and An, G. 2004. Rice functional genomics: large scale gene discovery and applications for crop improvement. Adv. Agron. 82: 55111.

M. Van Harten, "Mutation Breeding Theory and Practical Applications". Cambridge University Press, London, UK, 1998.

Manjunatha, G. A., Vanaja, T., Naik, J., Kumar, A. S. A. and Vasudevan, N. R. (2016) Identification of rice genotypes best suited for the development of organic varieties and identification of 
current varieties best suited for organic farming. J Organics. 3(1):16-24.

Mohapatra T, et al. EMS induced mutants of upland rice variety Nagina 22: generation and characterization. Proceedings of the Indian National Science Academy. 2004; 80(1):163172.

Mondal, B., Singh, S. P. and Joshi, D. C. (2014) DUS characterization of rice (Oryza sativa L.) using morphological descriptors and quality parameters. Outlook on Agric. 43:131-137.

Patra BC. 2000. Collection and characterization of rice genetic resources from Keonjhar district of Orissa. Oryza. 34:324-326.

PPV \& FRA. (2007) Guidelines for the conduct of test for Distinctiveness, Uniformity and Stability on rice (Oryza sativa L.). Pl Variety J India. 1:24- 25.

Rao, S. L. V., Prasad, G. S., Chiranjivi, M., Chaitanya, U. and Surendhar, R. (2013) DUS characterization for farmer varieties of rice. IOSR J Agric Vet Sci., 4:35-43.

Role of Mutation Breeding in Crop Improvement - Past, Present and Future Aamir Raina, Rafiul Amin Laskar, Shahnawaz Khursheed, Ruhul Amin, Younas Rasheed Tantray, Kouser Parveen 1 and Samiullah Khan Asian Research Journal of Agriculture 2(2): 1-13, 2016; Article no.ARJA.29334.

S. J. S. Rama Devi, Kuldeep Singh, B. Umakanth, B. Vishalakshi, K. Vijaya Sudhakara Rao, B. Suneel, S. K. Sharma, Gopala Krishna Murthy Kadambari, M. S. Prasad, P. Senguttvel, Divya P. Syamaladevi and M. S. Madhav, Identification and Characterization of a Large Effect QTL from Oryza glumaepatula Revealed Pi68(t) as Putative Candidate
Gene for Rice Blast Resistance https://doi.org/10.1186/s12284-02000378-4.

Sasaki A, et al. A mutant gibberellin synthesis gene in rice. Nature. 2002; 416(6882):701-702.

Singh Y and Singh US. 2008. Genetic diversity analysis in aromatic rice germplasm using agro morphological traits. Journal of Plant Genetic Resources. 21(1): 32-37.

Singh Yogendra (2011): Molecular approaches to assess genetic divergence in Rice. GERF Bulletin of Biosciences 02(01): 41-48.

Singh Yogendra and U.S Singh (2008): -Genetic diversity analysis in Aromatic Rice Germplasm using Agro- morphological Traits. Indian Journal of plant Genetic resources.21 (1) 32-37.

Singh Yogendra, Pani D.R, Khokhar D, Singh U S (2013). Agro-morphological Characterization and Molecular Diversity analysis of Aromatic Rice Germplasm Using RAPD Markers. Oryza.50 (01): 26-34.

Subodh K. Datta Asian Research Journal of Agriculture 2(2): 1-13, 2016; Article no.ARJA.29334 .

Suneel, P. Gopi, J. Karteek, M. D. Ershad, G. S. Laha, A. P. Padmakumari, R. M. Sundaram, V. Ramesh Sonti, H. K. Patel, P. Sudhakar, A. Krishna Satya and M. S. Madhav (2020) Assessment of Agro-morphological Characters among Complete Panicle Emergence Mutants of Samba Mahsuri (BPT5204) 39(10): 113-125, 2020; Article no.CJAST.56469.

Suprasanna, P., S. J. Mirajkar and S. G. Bhagwat. 2015. Induced mutations and crop improvement. In: Bahadur, B., V. M. Rajam, L. Sahijram and K. V. Krishnamurthy (Eds.), Plant Biology and Biotechnology. Vol. I. Plant 
Diversity, Organization, Function and Improvement, Springer India, pp. 593617.

Tirkey, A., Sarawgi, A. K. and Rao, S. L. V. (2013) Studies on genetic diversity in various qualitative and quantitative characters in rice germplasm. Ind $\mathbf{J}$ Plant Gen Res., 26:132-137.

Umarani, E., Radhika, K, Padma, V. and Rao, S. L. V. (2017) Agro-morphological characterization of rice (Oryza sativa L.) landraces based on DUS descriptors. Int J Pure App Biosci.,
5:466-475.

UPOV Guidelines for rice (Oryza sativa L.), TG 16/4. 1985; 2-18.

VRK Reddy. Cytogenetic effects of gamma rays and ethyl methane sulphonate in chilli pepper (Capsicum annuum L.). Cytologia. 2000; 65(2):129-133.

Y-P. Lai, J. Huang, J Li and Z-R. Wu, "A new approach to random mutagenesis in vitro", Biotechnology and Bioengineering, vol: 86 (6), p: 622627, 2004.

\section{How to cite this article:}

Suneel, B., G. S. Laha, A. P. Padmakumari, R. M. Sundaram, L. V. Subba Rao, P. Sudhakar, A. Krishna Satya and Madhav, M. S. 2020. Morphological and Molecular Characterization of Complete Panicle Emergence Mutant Lines for Assessing Genetic Relatedness. Int.J.Curr.Microbiol.App.Sci. 9(09): 549-561. doi: https://doi.org/10.20546/ijcmas.2020.909.070 